improve sustainability. As he pointed out, most people see plastics in a consumer context; they forget that plastics are ubiquitous. Polymeric materials must be considered in their entirety. This is where social sciences enter the mix. "The first thing that we have to do is to think about why, from a societal perspective, these things are important," Shaver said.

Take food packaging. With it, food is safer, lasts longer, and is protected from contamination. "We need to have people not die. At the same time as we prioritize what those plastics are doing from a societal perspective, we can look at what things we don't necessarily need," Shaver said. "What we need is for materials science to come in and innovate," he added. The biggest area of innovation now is the quest for "biosourced" and biodegradable materials. But then there is a real challenge in terms of marketing. Shaver warned that when people see the "biodegradable" label on packaging, they think that it will safely decompose wherever they dispose of it. In reality, many so-called biodegradable polymers require treatment in specialized facilities. "What we need is an infrastructure that supports industrial biodegradation," Shaver said. Is there any point in adopting these materials without that service? he asked.

"We, as materials experts, need to push back against the negative press around plastics," Shaver advised the materials experts at the meeting. According to Shaver, the materials community needs to prioritize systems for reuse and then recycling, and degradation and pyrolysis. "The most important thing to remember is that I cannot get a sustainable material, I can only get a sustainable system," he said. So, when pursuing sustainability, take on board the system approach before diving off into new materials, he added. Shaver also stressed the need for materials researchers to understand business models and social science practice as ways to "inform materials design." As Shaver put it, "sustainability is an overarching thing. So an initial framing now can help shape things in the future."

Michael Kenward

\section{EC awards emergency funds for COVID-19 research}

$\mathrm{O}_{\mathrm{r}}^{\mathrm{n}}$ January 30, 2020, the European Commission (EC) launched a "request for expressions of interest" to address the pandemic of the new coronavirus-induced disease called COVID-19. EC began with a budget of $€ 10$ million, which was subsequently increased up to $€ 48.5$ million. Such a rapid reaction was made possible by the standing budget line for emergency research funds that the EC maintains as part of the Horizon 2020 annual work programs for health research. This enabled 17 projects to be short-listed for funding, which the EC announced on March 6, 2020, to which an 18th project was added in late March. In total, these 18 projects involve 140 research teams across the EU, as well as non-European collaborators.

Projects that have been short-listed include rapid "point-of-care" diagnostic tests, treatment, and vaccines to combat COVID-19. Two approaches have been adopted for treatment. The first of these is to accelerate the development of new treatments currently in the pipeline (including therapeutic peptides, monoclonal antibodies, and broad-spectrum antivirals), and the second is to screen and identify molecules that could work against the virus, using advanced modeling and computational techniques. In the development of new vaccines, research will focus on developing a preventative vaccine and a therapeutic vaccine to be used for treatment.

For diagnostics, a project called CoNvat is developing advanced nanobiosensing platforms. Coordinated by Laura Lechuga of the Research Center on Nanoscience and Nanotechnology at the campus of Universitat Autònoma de Barcelona, the project includes researchers from Spain, Italy, and France. Their device, based on optical biosensor nanotechnology, is expected to become massively available in less than 12 months.

Another project, called CoronaDX, is spearheaded by Anders Wolff of the Technical University of Denmark. Among the devices the research group is developing is a portable instrument called PATHPOD. It contains a specially designed lab-on-achip, in which a full laboratory analysis is encoded into a microchip. The sample from a patient is placed on the chip, which analyzes by measuring the biological reactions, and the result is available on a computer or a tablet in 30 minutes. Wolff says, "In October [2020], we expect to have a permit for emergency use in Denmark and thus be ready for the possible second wave of coronavirus."
CoroNAb is the name of a project from Karolinska Institutet (KI) in Sweden, focused on developing antibodies that can block the virus' ability to infect cells, so-called neutralizing antibodies. In an interview the institute published, KI researcher Gerald McInerney, an associate professor of virology, said that his group cloned the spike protein based in the COVID-19 genetic code published in January. They have injected their spike protein into an alpaca, waiting to see if the animal develops antibodies against the protein.

APEPTICO, a privately held biotechnology company based in Austria, is developing peptide-based medicinal products to treat life-threatening pulmonary dysfunctions. The company has been selected to start the grant preparation to make its proprietary investigational medicinal product (IMP) solnatide available for medical treatment of patients severely affected by COVID-19. Solnatide is a synthetic molecule that is water-soluble and can be administered in aerosol form by oral inhalation. Solnatide is currently in Phase II clinical studies. For the EC project, APEPTICO has formed a consortium with companies from Germany, Italy, and The Netherlands to continue and speed up the manufacturing process of solnatide, as well as the immediate employment of solnatide IMP for clinical use. 